\title{
Smoke in the Pipe Nebula: dust emission and grain growth in the starless core FeSt 1-457
}

\author{
Jan Forbrich ${ }^{1,2}$, Charles J. Lada ${ }^{2}$, Marco Lombardi ${ }^{3}$, Carlos Román-Zúñiga ${ }^{4}$, and João Alves ${ }^{1}$ \\ 1 University of Vienna, Department of Astrophysics, Türkenschanzstraße 17, 1180 Vienna, Austria \\ e-mail: jan. forbrich@univie.ac . at \\ 2 Harvard-Smithsonian Center for Astrophysics, 60 Garden Street, Cambridge, MA 02138, USA \\ 3 University of Milan, Department of Physics, via Celoria 16, 20133 Milan, Italy \\ 4 Universidad Nacional Autónoma de México, Instituto de Astronomía, Ensenada BC 22860, Mexico
}

Received 20 November 2014 / Accepted 22 May 2015

\begin{abstract}
Context. The availability of submillimeter dust emission data in an unprecedented number of bands provides us with new opportunities to investigate the properties of interstellar dust in nearby clouds.

Aims. The nearby Pipe Nebula is an ideal laboratory to study starless cores. We here aim to characterize the dust properties of the FeSt 1-457 core, as well as the relation between the dust and the dense gas, using Herschel, Planck, 2MASS, ESO Very Large Telescope, APEX-Laboca, and IRAM 30 m data.

Methods. We derive maps of submillimeter dust optical depth and effective dust temperature from Herschel data that were calibrated against Planck. After calibration, we then fit a modified blackbody to the long-wavelength Herschel data, using the Planck-derived dust opacity spectral index $\beta$, derived on scales of $30^{\prime}$ (or $\sim 1 \mathrm{pc}$ ). We use this model to make predictions of the submillimeter flux density at $850 \mu \mathrm{m}$, and we compare these in turn with APEX-Laboca observations. Our method takes into account any additive zeropoint offsets between the Herschel/Planck and Laboca datasets. Additionally, we compare the dust emission with near-infrared extinction data, and we study the correlation of high-density-tracing $\mathrm{N}_{2} \mathrm{H}^{+}$emission with the coldest and densest dust in FeSt 1-457. Results. A comparison of the submillimeter dust optical depth and near-infrared extinction data reveals evidence for an increased submillimeter dust opacity at high column densities, interpreted as an indication of grain growth in the inner parts of the core. Additionally, a comparison of the Herschel dust model and the Laboca data reveals that the frequency dependence of the submillimeter opacity, described by the spectral index $\beta$, does not change. A single $\beta$ that is only slightly different from the Planck-derived value is sufficient to describe the data, $\beta=1.53 \pm 0.07$. We apply a similar analysis to Barnard 68 , a core with significantly lower column densities than FeSt 1-457, and we do not find evidence for grain growth but also a single $\beta$. Finally, our previously reported finding of a correlation of $\mathrm{N}_{2} \mathrm{H}^{+}$emission with lower effective dust temperatures is confirmed for FeSt 1-457 in mapping observations.

Conclusions. While we find evidence for grain growth from the dust opacity in FeSt 1-457, we find no evidence for significant variations in the dust opacity spectral index $\beta$ on scales $0.02<x<1 \mathrm{pc}$ (or $36^{\prime \prime}<x<30^{\prime}$ ). The correction to the Planck-derived dust $\beta$ that we find in both cases is on the order of the measurement error, not including any systematic errors, and it would thus be reasonable to directly apply the dust $\beta$ from the Planck all-sky dust model. As a corollary, reliable effective temperature maps can be derived which would be otherwise affected by $\beta$ variations. Finally, we note that the angular resolution of extinction maps for the study of nearby starless cores remains unsurpassed.
\end{abstract}

Key words. dust, extinction - stars: formation - submillimeter: ISM - infrared: ISM - radio lines: ISM

\section{Introduction}

The unprecedented wavelength coverage in the far-infrared and submillimeter range provided by Herschel and Planck has renewed interest in measuring and constraining the dust properties of the interstellar medium. However, while the data availability has improved tremendously, leading to much better-constrained spectral energy distributions (SED), such endeavours are still fundamentally limited by the degeneracy of the dust opacity and temperature in governing the shape of the SED and by the effect of line-of-sight averaging (e.g., Schnee et al. 2007; Shetty et al. 2009a,b; Kelly et al. 2012).

Briefly, the specific intensity of optically thin dust emission in the far-infrared and submillimeter wavelength range can be described as $I_{v}=B_{v}(T)\left[1-\mathrm{e}^{-\tau_{\nu}}\right] \approx B_{v}(T) \tau_{v}$ where $B_{v}(T)$ is the Planck function that describes blackbody radiation. The frequency dependence of the optical depth $\tau_{v}$ is typically described as $\tau_{v} \propto\left(v / v_{0}\right)^{\beta}$. Since $\tau_{v} \propto \kappa_{v} \Sigma_{\text {dust }}$, where $\kappa_{v}$ is the dust opacity and $\Sigma_{\text {dust }}$ is the dust column density, the spectral index $\beta$ also describes the frequency dependence of the dust opacity $\kappa_{v} \propto$ $\left(v / v_{0}\right)^{\beta}$.

In dense cores on scales of $\sim 0.1 \mathrm{pc}$, the dust opacity spectral index $\beta$ is expected to lie between $\beta=2$, as determined for the diffuse interstellar medium (e.g., Draine \& Lee 1984), and $\beta \sim 1$, as derived for circumstellar disks where grain growth has occurred (e.g., Beckwith \& Sargent 1991). In dense cores, dust coagulation and grain growth are two out of several processes that are thought to lower $\beta$ from the value that holds for the diffuse ISM. Ossenkopf \& Henning (1994) modeled the dust emission of protostellar cores and found $\beta \sim 1.8$.

Observational evidence for $\beta$ changes in low-mass starless cores has so far remained ambiguous - largely due to parameter degeneracies. Since these degeneracies are already difficult to disentangle for cores without an internal heating 
source, we restrict our discussion to starless cores. Kramer et al. (2003) and Bianchi et al. (2003), targeting IC 5146 and Barnard 68, respectively, compared ground-based bolometer data and infrared extinction data to constrain dust opacity ratios. They concluded that a dust opacity spectral index of $\beta \sim 2$ is compatible with their data. Shirley et al. (2005) used SCUBA data at $450 \mu \mathrm{m}$ and $850 \mu \mathrm{m}$ to derive $\beta=2.44 \pm 0.62$ for the low-mass starless core L1448, assuming a dust temperature of $10.5 \mathrm{~K}$ based on radiative transfer calculations. Schnee et al. (2010) observed the starless core TMC-1C with groundbased bolometers and Spitzer in five different bands; they derive $\beta=2.2 \pm 0.6$. While these measurements of $\beta$ indicate rather high values, the predictions of Draine \& Lee (1984) and Ossenkopf \& Henning (1994) cannot be ruled out within the uncertainties. Sadavoy et al. (2013) used Herschel and SCUBA-2 data to constrain dust properties in the Perseus B1 clump. Most recently, Schnee et al. (2014) used ground-based millimeter maps to constrain the dust properties of the entire OMC-2/3 region with both starless and protostellar cores. Assuming the dust temperature as equal to the measured kinetic temperature of ammonia, as derived from the $\mathrm{NH}_{3}(1,1)$ and $(2,2)$ transitions, they find a very low $\beta=0.9 \pm 0.3$ across the filament as well as the starless and protostellar cores.

As part of the Planck mission, an all-sky model of the Galactic foreground thermal dust emission has been developed; it has been described in detail in Planck Collaboration XI (2014). The model is based on submillimeter data collected at frequencies of 857,545 , and $353 \mathrm{GHz}$ in combination with IRAS $100 \mu \mathrm{m}$ data, and it has an angular resolution of $5^{\prime}$. The model takes into account three main parameters, i.e., the dust optical depth, the effective dust temperature, and the dust opacity spectral index $\beta$. The effective dust temperature is derived from a modified-blackbody fit toward each line of sight and thus does not directly correspond to a unique physical dust temperature. As explained by Planck Collaboration XI (2014), these parameters offer an empirical description of the data, even if their physical interpretation is complicated due to degeneracies and uncertainties.

After a careful study of parameter interdependencies and the impact of noise and cosmic infrared background anisotropies on the recovered parameters, Planck Collaboration XI (2014) decided to fit the data in a two-step process. In a first step, to minimize the impact of noise and the cosmic infrared background on the derivation of the dust opacity spectral index $\beta$ and the temperature, $\beta$ is derived on data that has been smoothed to a resolution of $30^{\prime}$. Then, in a second step, the temperature and opacity are fitted with a fixed $\beta$ at the nominal resolution of $5^{\prime}$. The underlying assumption here is that $\beta$ does not vary strongly on small scales. For the final data product, the typical uncertainty for the fit parameters is estimated at a few percent.

In order to study the relevance of this dust emission model on scales of individual cores, we here present a detailed observational study of the starless core FeSt 1-457 in the Pipe Nebula. Given its nearby location at a distance of $130 \mathrm{pc}$ (Lombardi et al. 2006), the Pipe Nebula is an ideal laboratory to study the physics of low-mass starless cores and the initial conditions of star formation (Lada et al. 2008; Rathborne et al. 2008; Forbrich et al. 2009, 2010). The FeSt 1-457 core has been previously targeted with millimeter line (Aguti et al. 2007; Frau et al. 2010; Forbrich et al. 2014), deep mid-infrared (Ascenso et al. 2013), and submillimeter polarization observations (Alves et al. 2014).

In the present study, we use submillimeter imaging and near-infrared extinction mapping to construct maps of the dust column density and the effective dust temperature toward FeSt 1-457. Additionally, we study the correlation of $\mathrm{N}_{2} \mathrm{H}^{+}$emission, as a molecular tracer of cold and dense gas, with the lowest measured effective dust temperatures. In Sect. 2, we describe the observations before we present the results in Sect. 3. The conclusions are presented in Sect. 4.

\section{Observations and data reduction}

\subsection{Herschel submillimeter continuum data}

Observations with the Herschel Spectral and Photometric Imaging Receiver (SPIRE) for this region were obtained on March 14, 2011, as part of a program described by Peretto et al. (2012). The SPIRE detector has three bands with center wavelengths 250, 350, and $500 \mu \mathrm{m}$, or center frequencies $1.2 \mathrm{THz}$, $857 \mathrm{GHz}$, and $600 \mathrm{GHz}$, respectively. These data were processed in combination with Planck data and 2MASS near-infrared extinction mapping as described by Lombardi et al. (2014) to obtain maps of dust optical depth and effective dust temperature at a better angular resolution than from Planck data alone. This methodology involves the calibration of Herschel data against the absolutely calibrated Planck data, and the use of 2MASS extinction maps to convert dust optical depth to column densities. Our analysis generally uses the largest Herschel beam size of $36^{\prime \prime}$, which is an improvement in angular resolution by a factor of $\sim 8$ when compared to the corresponding Planck data.

Following the procedure developed by Lombardi et al. (2014), we created maps of effective dust temperature from the calibrated Herschel data. To describe the dust emission along each line of sight and thus derive these effective dust temperatures, we have fitted a modified blackbody to the longwavelength Herschel SED, using a dust opacity spectral index $\beta$ fixed to the value determined in the Planck all-sky model. A single effective dust temperature, as a weighted average of temperatures along each line of sight, is sufficient to fit the data, but since the temperature can vary along the line of sight and still produce a well-behaved effective dust temperature, it does not mean that isothermal conditions are assumed. Due to these complications, the effective dust temperature does not directly correspond to a physical dust temperature of strictly isothermal dust even though it indirectly reflects a weighted average of such dust temperatures along the line of sight.

\subsection{APEX-Laboca submillimeter continuum data}

We additionally obtained ground-based submillimeter data of the FeSt 1-457 core and its surroundings with the Large APEX Bolometer Camera (Laboca). Laboca is a submillimeter bolometer array (Siringo et al. 2009) and a facility instrument of the Atacama Pathfinder Experiment (APEX) telescope (Güsten et al. 2006). The telescope is located on Llano de Chajnantor in the Chilean Andes, at an altitude of $5107 \mathrm{~m}$. The central frequency of the Laboca bolometers is $345 \mathrm{GHz}(870 \mu \mathrm{m})$, with a spectral passband that is about $60 \mathrm{GHz}$, or $150 \mu \mathrm{m}$, wide $(F W H M)$. Observations were carried out as part of our project 84.C-0927 on 23 and 24 September 2009. The FeSt 1-457 core was mapped for about 35 minutes with fluxes calibrated against the primary calibrator Neptune (and G5.89). A combination of spiral maps and on-the-fly maps was used to cover the area. At the Laboca central wavelength, the beam size of the APEX telescope is $19^{\prime \prime} F W H M$. The data have been processed according to the iterative procedure described by Belloche et al. (2011) to maximize the signal-to-noise ratio. The final noise level from combining the individual maps is $11 \mathrm{mJy} /$ beam. In our analysis, 

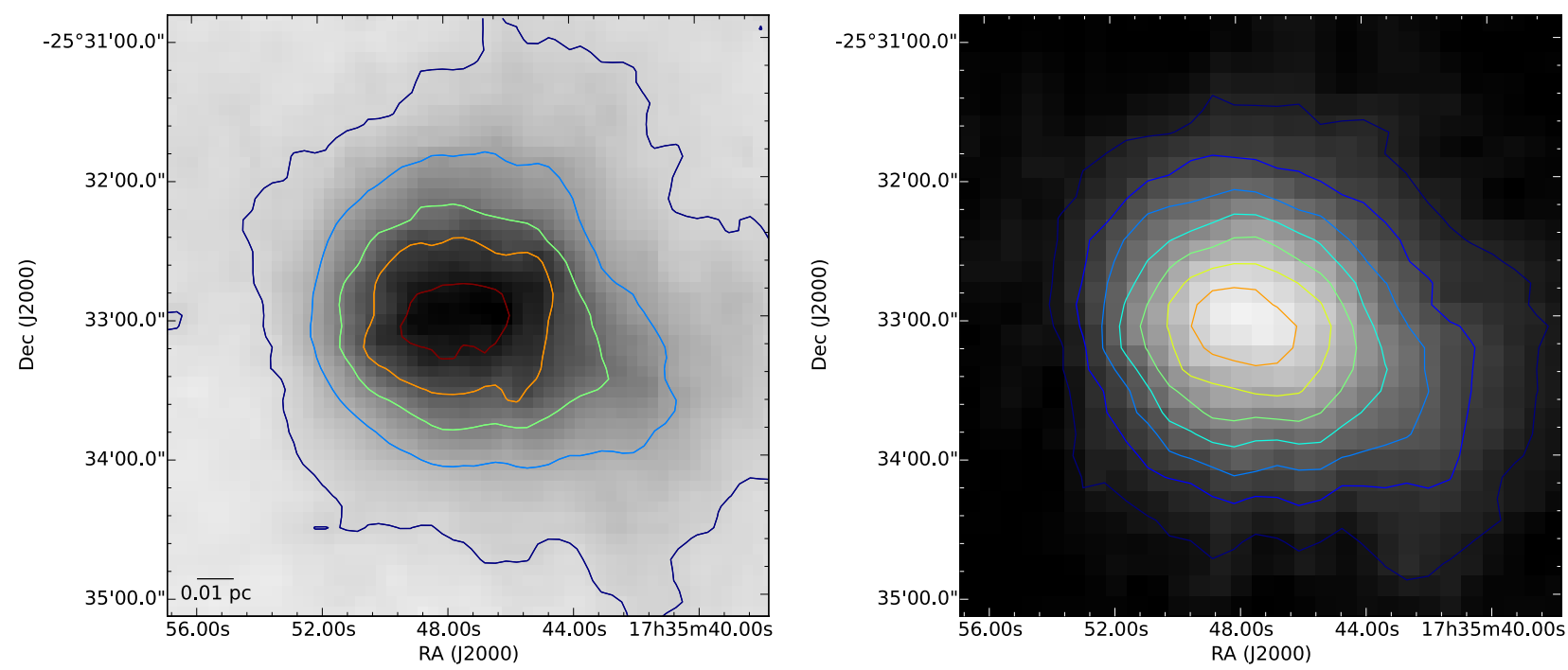

Fig. 1. Left: near-infrared extinction map, resolution: $9^{\prime \prime} F W H M$. Contours indicate levels of $A_{K}=1$ to 5 mag, in steps of 1 mag. Right: Laboca $870 \mu \mathrm{m}$ map, $18^{\prime \prime} F W H M$. Contours indicate levels of $0.033 \mathrm{Jy} /$ beam, corresponding to the $3 \sigma$ limit, to $0.231 \mathrm{Jy} /$ beam, in steps of $0.033 \mathrm{Jy} / \mathrm{beam}$.

we take into account the theoretical instrument spectral response as published in Siringo et al. (2009) and on the APEX website ${ }^{1}$.

\subsection{Near-infrared data}

In addition to the 2MASS data that are used as part of the Herschel calilbration procedure described above, we use deeper near-infrared data to obtain an even better extinction map of FeSt 1-457. Since its angular resolution exceeds that of Herschel and Laboca, this map serves as a reference comparison for both. The near-infrared ground-based observations used in this context are part of a high-resolution near-infrared survey of regions across the whole Pipe Nebula (Román-Zúñiga et al. 2009, 2010). The data for the Fest 1-457 core come from two different datasets. The first set is of $H$ and $K_{\mathrm{s}}$ images obtained with the Son of ISAAC (SofI) camera at the ESO New Technology Telescope (NTT) atop Cerro La Silla in Chile. The resolution of the SofI-NTT observations is 0.'288. The second set of ground-based data are $H$ and $K_{\mathrm{s}}$ images obtained with the Infrared Spectrometer And Array Camera (ISAAC) at the ESO Very Large Telescope (VLT) atop Cerro Paranal in Chile. These ISAAC-VLT observations were carried out during 2002 July. The VLT observations targeted the areas of peak extinction of the core and its nucleus. The high angular resolution ( 0 .' 148$)^{\prime}$ and sensitivity of these observations allowed us to detect several highly reddened background sources that could not be detected with SofI, allowing us to construct a complete NICEST extinction map (Lombardi 2009) of the core at a resolution of $9^{\prime \prime}(F W H M)$. At this resolution and a pixel size of $4 . .5$, it is still possible to ensure the presence of a minimum of about ten (and often many more) background stars per resolution element (beam) for this method to work.

\subsection{Millimeter line data}

Observations in $\mathrm{N}_{2} \mathrm{H}^{+}(1-0)$ and other transitions were obtained with the IRAM $30 \mathrm{~m}$ telescope in July 2003. These observations have been reported and described in detail by Aguti et al. (2007),

\footnotetext{
1 http://www . apex-telescope.org/bolometer/laboca/ technical/
}

and we here use their $\mathrm{N}_{2} \mathrm{H}^{+}(1-0)$ map to compare this tracer of cold and dense gas with our effective dust temperature map.

\section{Results and analysis}

For a general overview of the FeSt 1-457 core, we show the high-resolution extinction map $\left(9^{\prime \prime} F W H M\right)$ and the Laboca dust emission map (18" FWHM) in Fig. 1. The Herschel-based effective dust temperature and dust optical depth maps are shown in Fig. 2. We use the best available angular resolution wherever possible, but to compare all three datasets, we use a common resolution of $36^{\prime \prime}$, reached by convolving the higher-resolution data to larger beam sizes. At a distance of $130 \mathrm{pc}$, an angular size of $36^{\prime \prime}$ corresponds to a scale of $0.02 \mathrm{pc}$. For intercomparisons, the extinction and Laboca data were thus convolved to this lower resolution.

In the following, we first use the Herschel SED fit and resulting dust model to demonstrate that the FeSt 1-457 core is not isothermal. Then, we compare the Herschel-derived dust optical depth with the independently derived near-infrared extinction map to constrain the dust opacity $\kappa_{v}$. Finally, we use the Herschel dust model to predict the submillimeter flux density observed by Laboca. Confronting this prediction with the observations allows us to constrain the dust opacity spectral index $\beta$.

\subsection{Temperature profile}

At a resolution of 36 " FWHM, the analysis of the Herschel effective dust temperature and $353 \mathrm{GHz}$ dust optical depth, as derived according to Lombardi et al. (2014), reveals significant gradients in both quantities and a tight anticorrelation, shown in Fig. 3. The FeSt 1-457 core shows a clear decrease in effective dust temperature toward its center. A plot of the same temperature data versus radius of the core is shown in Fig. 4. The lowest effective dust temperature at the center of the core is $13.50 \pm 0.05 \mathrm{~K}$. This is $4 \mathrm{~K}$ higher than the kinetic temperature of $T_{K}=9.5 \pm 0.1 \mathrm{~K}$ derived from dense-gas-tracing $\mathrm{NH}_{3}$ observations obtained with the Green Bank Telescope and a corresponding beam size of $30^{\prime \prime}$ (Rathborne et al. 2008).

However, in the Herschel data, we do not only trace the gas and dust at the center of the core. Instead, we always observe 

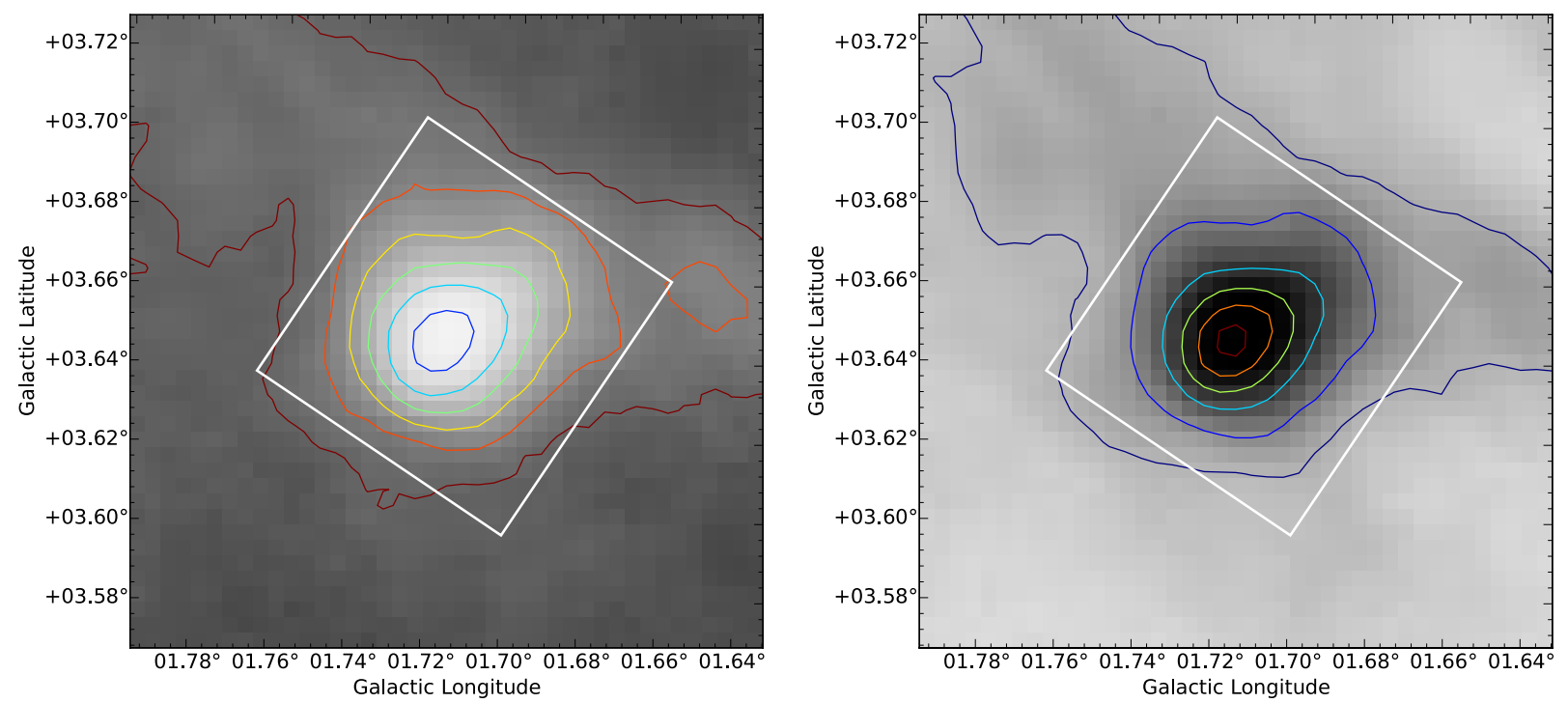

Fig. 2. Left: effective dust temperature map of FeSt 1-457, 36" FWHM, with contours indicating temperatures of $20 \mathrm{~K}$ down to $14 \mathrm{~K}$ in steps of $1 \mathrm{~K}$. Right: corresponding optical depth map, with contours indicating levels of 2, 3, 5, 7, 9, and $11 \times 10^{-4}$. In both panels, the white box indicates the field of view of Fig. 1 .

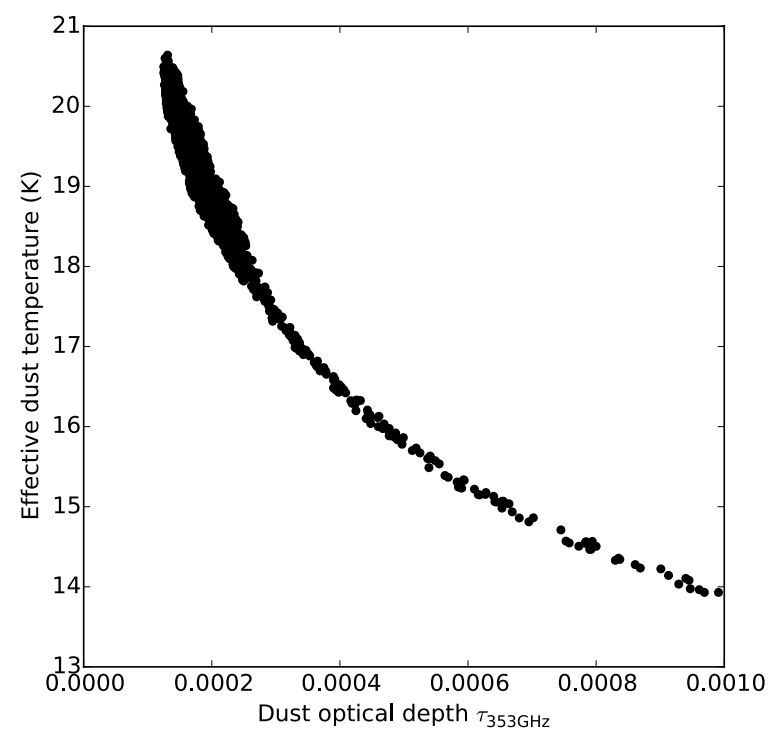

Fig. 3. Correlation of the submillimeter dust optical depth (at $353 \mathrm{GHz}$ ) and the effective dust temperature, as derived using the method described by Lombardi et al. (2014), at a resolution of 36" FWHM.

the entire line of sight through the core with its entire temperature structure. The effective dust temperature, as a weighted average along the line of sight derived from an SED fit, is our main single observable of the temperature conditions in the core. Similarly, when we use the same SED model to predict the flux density as observed with Laboca, it is the same effective dust temperature that enters the Planck function consistently. Deconvolving the actual absolute temperature profile from the observed effective dust temperature profile would require detailed modeling of the core which is beyond the scope of this paper.

If the density is sufficiently high, we would expect the gas and dust to be thermodynamically coupled such that the dust temperature and the gas kinetic temperature would be the same, but it is not obvious whether and where that would be the case in the FeSt 1-457 core. Assuming that the $\mathrm{NH}_{3}$ kinetic temperature

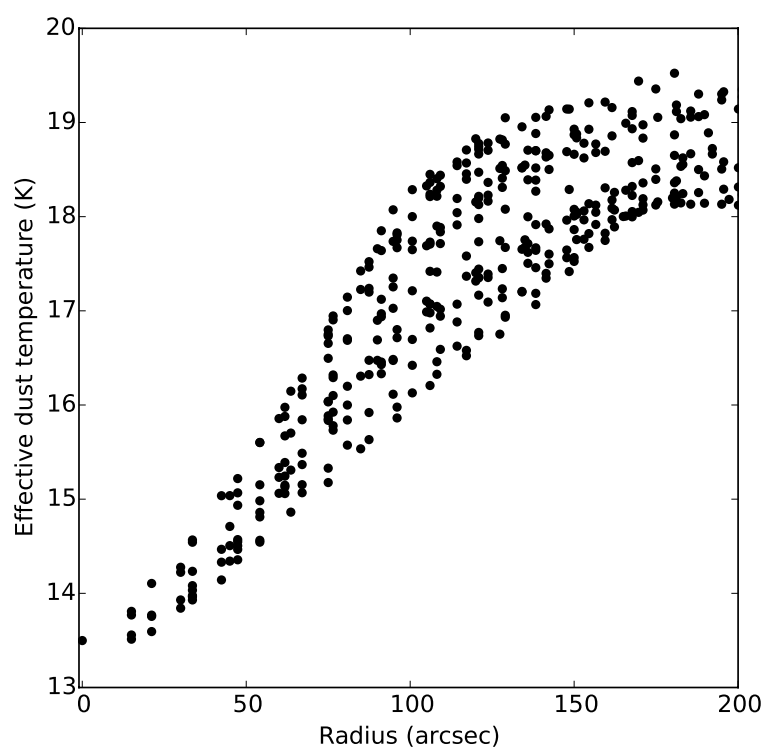

Fig. 4. Effective dust temperature toward the FeSt 1-457 core, as a function of core radius.

is a good approximation of the dust temperature at the center of the core would indicate that the effective dust temperature toward the center overestimates the actual dust temperature at the center by about $4 \mathrm{~K}$ which is similar to findings reported for Barnard 68 (see Sect. 3.6).

We can shed additional light on the gas-to-dust coupling by comparing the effective dust temperatures to molecular emission of $\mathrm{N}_{2} \mathrm{H}^{+}$as a tracer of high volume densities. When studying a large sample of Pipe cores, including the FeSt 1-457 core, Forbrich et al. (2014) noted a correlation of $\mathrm{N}_{2} \mathrm{H}^{+}(1-0)$ detections with low effective dust temperatures. Out of 52 cores, only the coldest six, with effective dust temperatures between 13.2 and $14.6 \mathrm{~K}$ were detected in $\mathrm{N}_{2} \mathrm{H}^{+}$emission. While that study only contained a single pointing at the FeSt $1-457$ core (source 96), it was among the $\mathrm{N}_{2} \mathrm{H}^{+}$detections. 


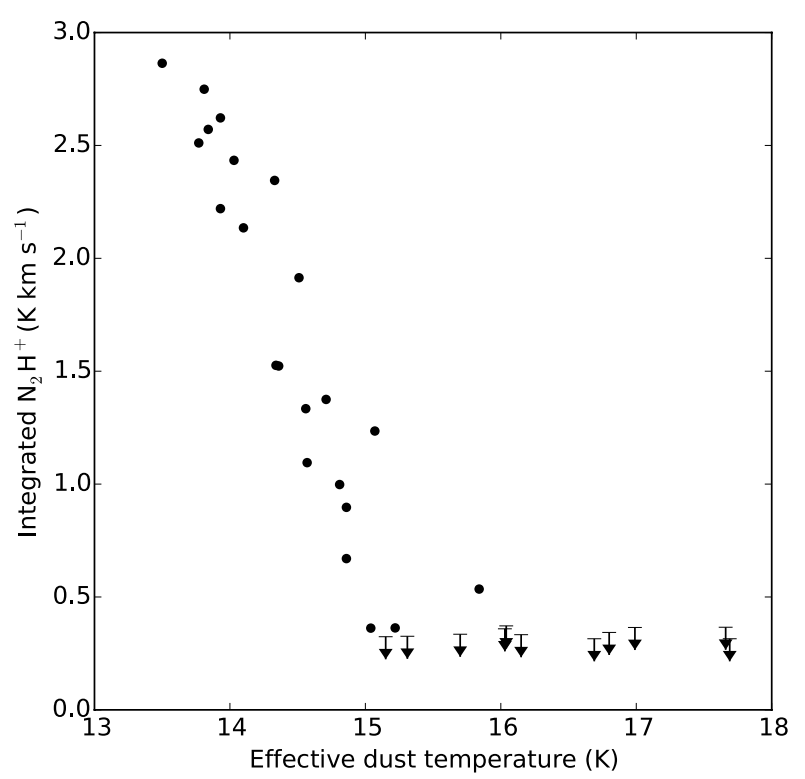

Fig. 5. Integrated $\mathrm{N}_{2} \mathrm{H}^{+}$emission (including all HFS components) vs. effective dust temperature in the FeSt 1-457 core. Arrows denote $3 \sigma$ upper limits. The emission is clearly correlated with the coldest temperatures.

We can now look at the correlation of effective dust temperatures and $\mathrm{N}_{2} \mathrm{H}^{+}$emission in more detail by making use of the $\mathrm{N}_{2} \mathrm{H}^{+}(1-0)$ map of FeSt 1-457 that was obtained by Aguti et al. (2007). Using the IRAM $30 \mathrm{~m}$ telescope, they reach an angular resolution of $26^{\prime \prime} F W H M$. We use the same data that have been described in Aguti et al. (2007), and we simply integrate the spectra over velocity intervals that cover the $\mathrm{N}_{2} \mathrm{H}^{+}(1-0)$ hyperfine structure components. To approximately compare with the Herschel effective dust temperatures, we have read out the closest pixel from the column density map with $36^{\prime \prime}$ FWHM; the pixel size is $15^{\prime \prime}$. The result is shown in Fig. 5. The strongest emission correlates with the lowest effective dust temperature, and there is a steep fall-off with rising temperatures, similar to the temperature range with $\mathrm{N}_{2} \mathrm{H}^{+}$detections that was reported by Forbrich et al. (2014). Interestingly, the correlation of $\mathrm{N}_{2} \mathrm{H}^{+}$ emission and low effective dust temperatures thus is not only seen in a sample of single observations of many different cores, but also in a map of a single core. Even as a weighted average along the line of sight, the effective dust temperatures can serve as a means of identification of the cold and dense conditions inside molecular cores.

\subsection{The dust opacity $\kappa_{v}$}

A comparison of the dust optical depth $\tau$ with the independently derived near-infrared extinction yields a contraint on changes of the dust opacity $\kappa_{v}$. When studying regions of comparably low extinction $\left(A_{K}<2 \mathrm{mag}\right)$, Lombardi et al. (2014) find a linear relation $A_{K}=\gamma \tau_{353 \mathrm{GHz}}+\delta$ where $\gamma$ is proportional to the ratio of the near-infrared extinction coefficient at $2.2 \mu \mathrm{m}$ and the submillimeter dust opacity $\kappa_{353} \mathrm{GHz}, \gamma \propto C_{2.2} / \kappa_{353} \mathrm{GHz}$. In our case, the slope of $\tau_{353 \mathrm{GHz}}\left(A_{K}\right)$ is proportional to $1 / \gamma$.

In Fig. 6, we show a comparison of the dust optical depth and an extinction map, produced with matching 36" FWHM resolution. The correlation between the two quantities is clearly nonlinear. For reference, we show a linear fit to the lowextinction part of the plot, only taking into account points with $A_{K}<2$ mag. Toward the highest near-infrared extinctions, above

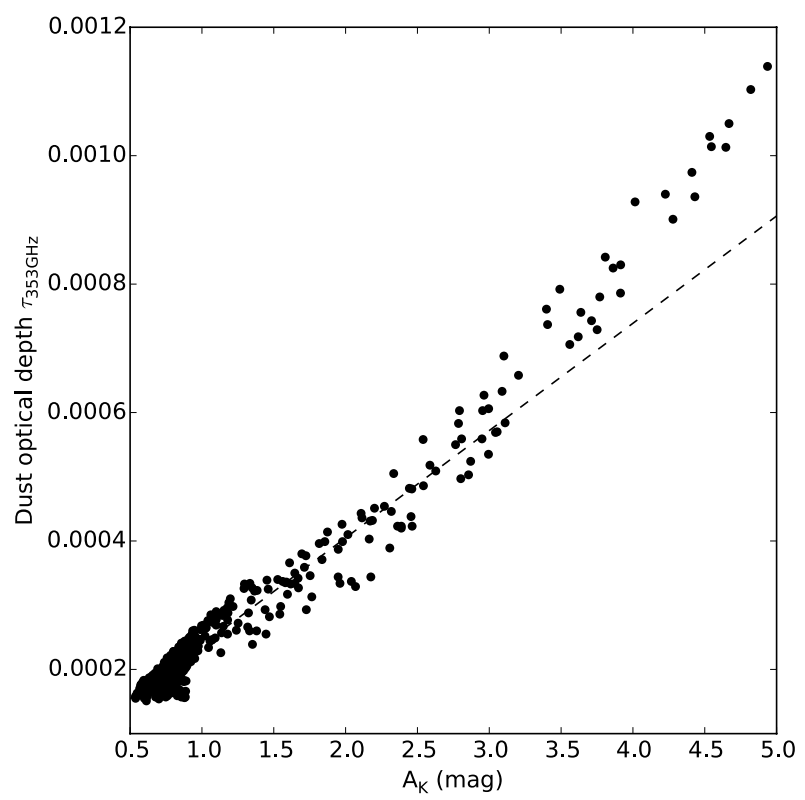

Fig. 6. Comparison of dust optical depth $\tau_{353 \mathrm{GHz}}$ and near-infrared extinction $A_{K}$. The dashed line shows a linear fit of the points with $A_{K}<2$ mag.

approximately $A_{K} \sim 3 \mathrm{mag}$, the submillimeter dust optical depth $\tau_{353 \mathrm{GHz}}$ rises faster than predicted from this low-extinction linear fit. To stay in the picture introduced by Lombardi et al. (2014), this means that $1 / \gamma$ increases with extinction.

An increase of $1 / \gamma$ toward the highest levels of extinction, i.e., an increase toward the center of the core, means that either the submillimeter dust opacity $\tau_{353 \mathrm{GHz}}$ is increasing or the nearinfrared extinction coefficient is decreasing, or both. If grain growth occurs in the central regions of this core, this would exactly be the expected observational signature (e.g., Ormel et al. 2011). In a study of the mid-infrared extinction law of the same core, Ascenso et al. (2013) had found evidence for large grains, but throughout the entire core and not just its innermost regions. Since the sensitivity to given grain sizes is different between these various methods, the finding of Ascenso et al. (2013) may well be compatible with our finding. In combination, this may mean that in the inner core, it is mostly the submillimeter dust opacity that is changing and not the infrared extinction coefficient.

We can make a simple estimate of the volume densities occurring in the central regions of the FeSt 1-457 core by determining the average volume density along the line of sight under the assumption of spherical symmetry. The peak extinction is $A_{K}=4.88 \mathrm{mag}$ on a size scale of $0.02 \mathrm{pc}$. Assuming an approximate conversion of $N_{H} \sim 2 \times 10^{22} A_{K} \mathrm{~cm}^{-2}$ (Ryter 1996; Vuong et al. 2003), this extinction corresponds to a column density of $9.76 \times 10^{22} \mathrm{~cm}^{-2}$. Given a projected extent in the plane of the sky of $<240^{\prime \prime}$ or $0.15 \mathrm{pc}$, this column density corresponds to an average volume density of $2.1 \times 10^{5} \mathrm{~cm}^{-3}$. This is a lower limit for the volume density at the center of the core but it is nevertheless an estimate for the volume densities where we find evidence for grain growth.

\subsection{The dust opacity spectral index $\beta$}

In addition to the Herschel dust model and the near-infrared extinction map, we can now use the Laboca submillimeter map to derive further constraints on the dust emission. We argue that 


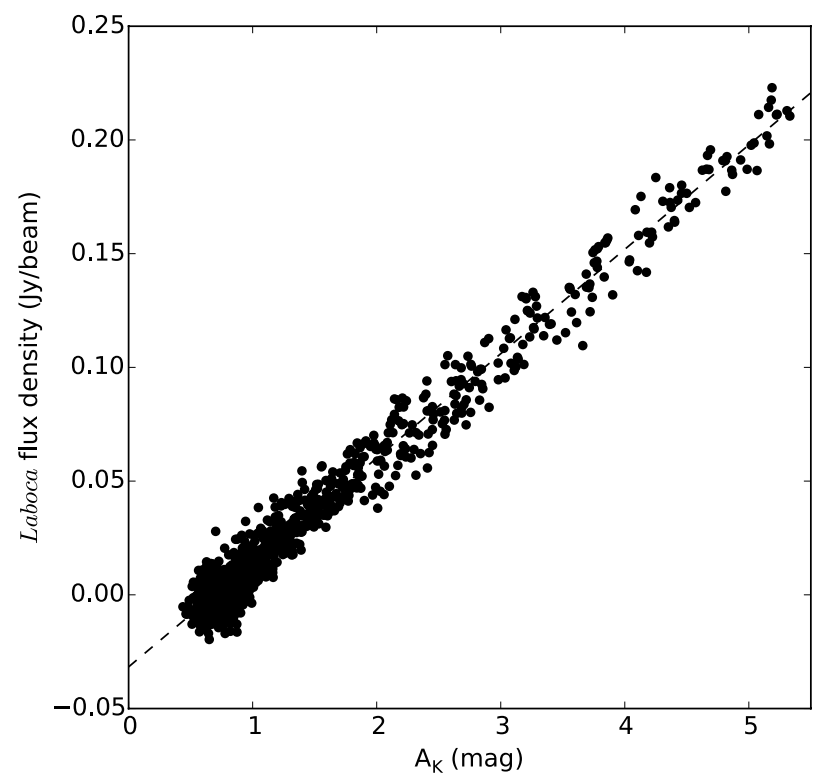

Fig. 7. Comparison of the Laboca map and a NICEST near-infrared extinction map with matching resolution (18" FWHM). The dashed line shows a linear fit above $A_{K}>1$ mag to avoid noisy points.

in particular it is possible to constrain the dust opacity spectral index $\beta$.

First of all, in a measurement that is entirely independent of the Herschel dust model, we find a linear trend of the Laboca submillimeter flux density as a function of near-infrared extinction $A_{K}$. In Fig. 7 we show this trend at the native Laboca resolution of $18^{\prime \prime} F W H M$, using an extinction map with the same resolution. The dashed line shows a simple linear fit $(f(x)=a x+b)$ to the data where $a=0.0459 \pm 0.0003$ and $b=-0.0317 \pm 0.0008 \mathrm{Jy} /$ beam. A zeropoint offset becomes apparent that we will quantify and discuss below for data convolved to $36^{\prime \prime} F W H M$. To within the errors, the comparison of Laboca submillimeter emission and near-infrared extinction reveals a linear correlation that holds up to the highest extinction levels. This observational result is surprising since, as we will discuss now, the flux density depends on both the dust optical depth and the temperature, and both are nonlinear functions of $A_{K}$, as we have seen.

It turns out that Laboca allows us to separately probe the region for evidence of a change in the dust opacity spectral index $\beta$. For this purpose, we use the Herschel dust model to predict the flux density observed by Laboca to then confront this prediction with the observations. To match the resolution of the dust model, the Laboca data have been convolved to match the resolution of the Herschel data at $36^{\prime \prime}$ FWHM.

Assuming optically thin submillimeter dust emission, which is a safe assumption judging by the Herschel data, the predicted Laboca flux density for every line of sight depends on the effective dust temperature, the dust optical depth $\tau_{v}$, and the dust optical depth (and opacity) spectral index $\beta: S_{v}=B_{v}(T) \cdot(1-$ $\left.\mathrm{e}^{-\tau_{\nu}}\right) \cdot \Omega_{\mathrm{b}} \cdot 10^{26} \mathrm{Jy} /$ beam, where $B_{v}(T)$ is the Planck function and $\Omega_{\mathrm{b}}$ is the beam solid angle. To take into account the Laboca spectral response, described in Sect. 2.2, we determine $S_{v}$ for a list of frequencies across the Laboca spectral response and weigh these individual flux densities into a total flux density according to their relative weights. Note that the Laboca fluxes are implicitly calibrated against the spectrum of Neptune with a spectral slope of about $\beta_{\text {planetary }} \sim 2$, which is close to the spectral slopes

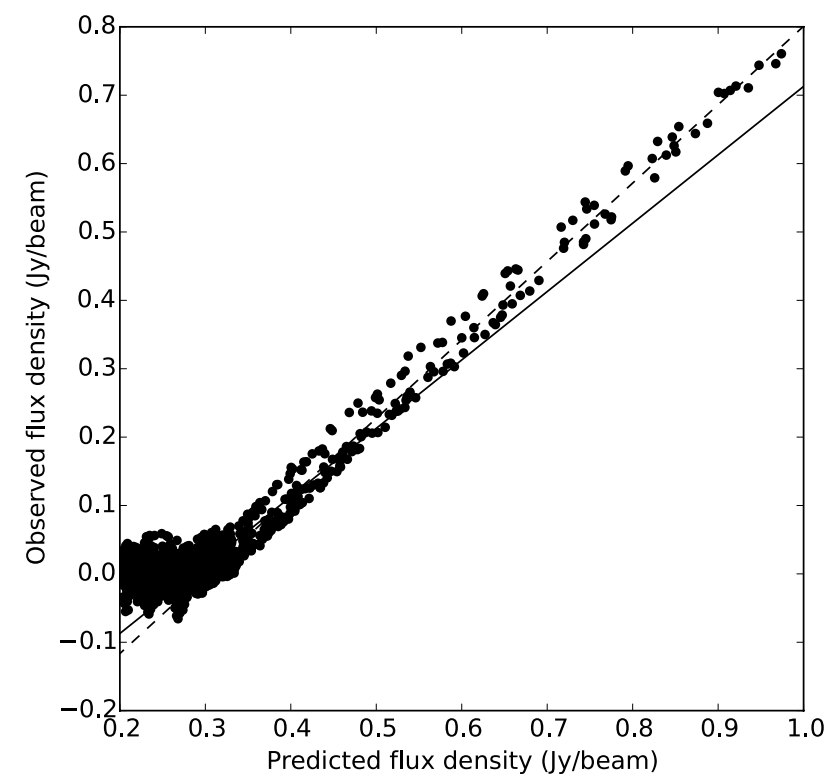

Fig. 8. Comparison of the observed submillimeter flux density from Laboca and the predicted flux density based on the Herschel/Planck model. The dashed line shows a linear fit of all points with a predicted flux density of $>0.4 \mathrm{Jy} / \mathrm{beam}$. For comparison, the continuous line indicates the reference slope of 1 (arbitrarily set to intersect with the fit at its starting point of $x=0.4 \mathrm{Jy} /$ beam) which would correspond to $\beta=\beta_{\text {Planck. The difference in slope corresponds to a difference of }}$ $\Delta \beta=0.15$ (see text).

discussed here, so that no color correction is applied (see also Planck Collaboration IX 2014). Such a correction would be by less than one percent in flux density which is an accuracy anyway not reached by ground-based submillimeter measurements.

The only two variables in this prediction are the temperature $T$ and the dust opacity spectral index $\beta$, used to calculate the dust optical depth for every frequency in the Laboca pass band, starting from $\tau_{353 \mathrm{GHz}}$, where the reference frequency already lies inside the Laboca pass band. For these two variables, $T$ and $\beta$, we use the effective dust temperature and the dust opacity spectral index from the Planck dust model at this position, $\beta_{\text {Planck }}=1.68 \pm 0.06$. While the effective dust temperature has already been derived from a Planck function, the choice of the Planck- $\beta$ is less obvious even if there is no alternative due to the lack of other constraints. In the Herschel dust model, the Planck- $\beta$ has already been used to determine $\tau_{353 \mathrm{GHz}}$, extrapolating from a frequency near the peak of the SED (here assumed to be $857 \mathrm{GHz}$ or $350 \mu \mathrm{m})$. Since the Planck- $\beta$ has been derived on scales much larger than the core $\left(30^{\prime}\right.$, or $1.1 \mathrm{pc}$ at a distance of $130 \mathrm{pc}$ ), its relevance for the description of the dust emission on (sub-)core scales is a priori unclear.

In Fig. 8, we directly compare the submillimeter flux density, as observed with Laboca, with the flux density that is predicted based on the Herschel dust model. The direct comparison of observed vs. predicted flux density, taking into account the temperature profile of the core in particular, results in a linear relation. There is an obvious zeropoint offset between the observed and predicted flux densities, which we will discuss below. Also, the systematic pattern of points in the plot, which look like two separate branches of the correlation, can be explained by a subpixel relative shift in astrometry between the Herschel/Planck and Laboca maps which cannot be verified independently. The slope of the relation is close to one, which is remarkable, given 
that the local dust $\beta$ may have nothing to do with the one derived from Planck data on larger scales of 30'.

According to a linear fit, the Laboca flux density at the Herschel/Planck zeropoint is $-0.346 \pm 0.002 \mathrm{Jy} / \mathrm{beam}$. The fit result for the slope is $a=1.147 \pm 0.006$ up to the highest flux densities, corresponding to the highest column densities. This means that a single dust $\beta$ is sufficient to describe these data. However, the fact that the slope is different from one indicates that the true value of $\beta$ is slightly different from $\beta_{\text {Planck. }}$. Since the dust model is able to predict the observed submillimeter flux densities, apart from zeropoint offsets, this result also means that changes in the dust optical depth and the effective dust temperature partly cancel out in the observed flux density to produce a linear relation of flux density vs. extinction.

In order to test whether the Laboca measurements can be explained by a single but potentially different $\beta_{\mathrm{N}} \neq \beta_{\text {Planck }}$ we first use $\beta_{\text {Planck }}$ to obtain the optical depth at a Herschel frequency near the peak of the SED (here assumed to be $857 \mathrm{GHz}$ or $350 \mu \mathrm{m}$ ) by extrapolation from $\tau_{353 \mathrm{GHz}}$. Then we introduce a different $\beta_{\mathrm{N}}$ as a variable to extrapolate back to the Laboca observing frequency, such that the combined application yields the measured Laboca flux density. The ratio of measured to predicted flux density then is:

$$
\frac{S_{\text {Laboca }}}{S_{\text {predicted }}}=\left(\frac{857 \mathrm{GHz}}{353 \mathrm{GHz}}\right)^{\beta_{\text {Planck }}-\beta_{\mathrm{N}}} \text {. }
$$

The slope of the linear function in Fig. 8 thus is a direct measure of the $\Delta \beta=\beta_{\text {Planck }}-\beta_{\mathrm{N}}$, where $\beta_{\mathrm{N}}$ provides a better description of the data: a ratio of the observed vs. predicted flux densities yields $(857 / 353)^{\Delta \beta}=a$. We then find $\Delta \beta=\ln (a) / \ln (857 / 353)=0.15 \pm$ 0.01 where the error estimate merely propagates the error in $a$ from the fit. The result is that a dust $\beta$ that is close to the Planck value can describe the data: $\beta_{\mathrm{N}}=1.68-0.15=1.53 \pm 0.07$. In fact, $\beta_{\mathrm{N}}$ is compatible with the Planck result to within the nominal respective $2 \sigma$ errors. It may be worth pointing out that even the use of the improved $\beta_{\mathrm{N}}$ results in an underprediction of the highest observed flux densities, i.e., those at the center of the core.

The advantage of this estimate is that it does not depend on zeropoint offset that is clearly present in the data shown in Fig. 8. Two effects contribute to this zeropoint offset: any actual calibration offset between the two datasets and spatial filtering of large-scale emission in the Laboca data. Additionally, the fact that the relation of measured to predicted flux density is entirely linear with a zeropoint offset suggests that spatial filtering in the Laboca data due to correlated-noise removal does not set in at angular scales that would affect the core. Belloche et al. (2011) show that the ratio of output to input flux density due to spatial filtering in Laboca data for a Gaussian source of size 200" (FWHM) is still $90 \%$. Our source FeSt 1-457 is smaller than this size, and the spatial filtering in this case only affects a constant background flux density, corresponding to the largest angular scales. Indeed, the Laboca map falls to zero flux density outside of the core while the Herschel/Planck map does not. We thus have a situation where a zeropoint offset is caused both by a possible calibration offset and spatial filtering on the largest scales.

Alternatively, to reconcile the Laboca measurements and the Herschel/Planck prediction, we could leave the dust $\beta$ unchanged and instead modify the effective dust temperatures to match the predicted and observed flux densities. Even if in reality both the dust opacity spectral index $\beta$ and the temperatures differ from our assumptions, changing either one of the two provides us with two instructive thought experiments.
After concluding that a single dust opacity spectral index $\beta_{\mathrm{N}}$ that is only slightly different from the Planck-derived value can reconcile the Laboca and Herschel/Planck datasets, we now solve $S_{v}=B_{v}\left(T_{\mathrm{d}}\right) \tau_{\mathrm{d}} \Omega$ for the dust temperature $T_{\mathrm{d}}$ while requiring the submillimeter flux density to match the observed value. In this experiment, it is mandatory to take into account the zeropoint offset. If both the different slope and the offset are to be explained by temperature effects, the result is an unphysical temperature profile for a starless core where the temperature would rise toward the center. Since we know that the Laboca measurements may have an effective zeropoint offset due to spatial filtering, we solve for a temperature prediction after adding $0.346 \mathrm{Jy} /$ beam to every pixel, as derived from the linear fit discussed above.

Without the zeropoint offset, generally higher effective dust temperatures could account for the difference between the measured and predicted Laboca fluxes. The temperatures change in nonlinear fashion, and the scatter for a given core radius increases considerably (not shown). While this scenario of individually modified temperatures cannot be ruled out, we prefer the scenario with a single, if slightly modified dust- $\beta$ because of its simplicity. Higher temperatures do not appear to be plausible since the line-of-sight weighted averages of the dust temperature are already an overestimate of the local dust temperature in the inner core.

In the light of the recently reported results of Schnee et al. (2014), we note that we have also tried to emulate their experiment for the case of FeSt 1-457. However, we do not have a map of the gas kinetic temperature of $\mathrm{NH}_{3}$, but only a single measurement toward the center of the core, as mentioned above. As a zero-order approximation, we have subtracted the difference between the minimum effective dust temperature and the ammonia gas kinetic temperature, a difference of $4 \mathrm{~K}$, from all points of our effective temperature map, thereby forcing the observed dust temperature toward the center of the core to be equal to the ammonia gas kinetic temperature while keeping the temperature structure of the map. We find that repeating the above experiment then requires a very different $\beta=0.64$ to explain the data. While this value would be within the range reported by Schnee et al. (2014), it would be very different from the Planck value. However, given that we know that a temperature gradient is present in such cores and given the degeneracy of $\beta$ and $T$ in the observations and its impact on the effective dust temperature as an observable, as well as uncertainties as to when the gas and dust can be assumed to be fully thermodynamically coupled, it is not obvious that any gas kinetic temperature can directly be used as a physical dust temperature in the line of argument described above.

\subsection{Zeropoint offsets}

This leaves the zeropoint offset between Herschel/Planck and Laboca to be explained. As determined from the fit, the Laboca flux density at the Herschel/Planck zeropoint is $-0.346 \pm$ $0.002 \mathrm{Jy} /$ beam. As noted above, the most obvious reason for this offset lies in the spatial filtering of large-scale structure, larger than the FeSt 1-457 core. Indeed, at a distance of about 2.5 from the core, the Laboca flux density falls to zero while the Planck never reach zero in the neighborhood of the FeSt 1-457 core. We directly compare the Laboca map to the Planck image at $353 \mathrm{GHz}$, i.e., at essentially the same observing frequency as Laboca. With a beam size of about 4!.8 FWHM, the Planck data have lower angular resolution compared to Laboca, but they cover the entire area. Selecting an image of $17^{\prime} \times 17^{\prime}$ (containing 

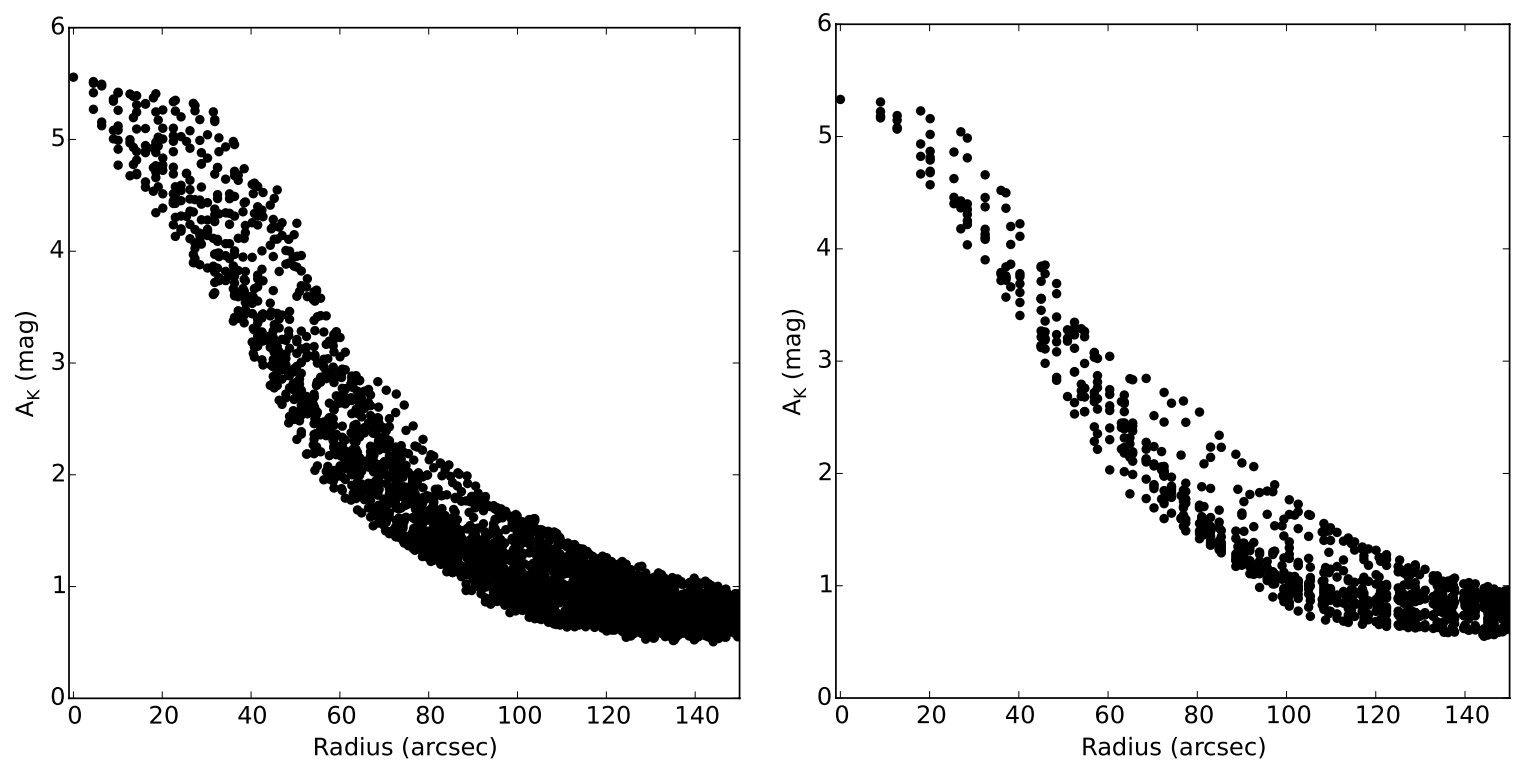

Fig. 9. Left: column density profile of the FeSt 1-457 core, using an extinction map with 9" resolution and 4".5 pixels. Right: column density profile of the FeSt 1-457 core, using an extinction map with $18^{\prime \prime}$ resolution and 9" pixels.

22.1 FWHM beam areas), the contrast of the peak, at the position of the core, to the mean pixel value of the image is only 1.38. If we assume that this contrast represents the large-scale emission, this would mean that at the peak of the Laboca image, where a flux density of $0.77 \mathrm{Jy} /$ beam $\left(36^{\prime \prime} F W H M\right)$ is measured, extended emission of $0.29 \mathrm{Jy} /$ beam has been filtered out which is very close to the observed zeropoint offset.

This suggests that a large fraction of extended emission, unrelated to the core, is filtered out by the Laboca imaging, but it is present in the Planck data. The Planck point source catalog provides us with an additional means to judge the spatial filtering. The Planck Catalog of Compact Sources Release 1 (Planck Collaboration XXVIII 2014) lists the FeSt 1-457 core

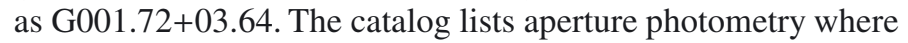
the aperture has a radius equal to the FWHM beam size and an adjacent annulus for local background subtraction. The annulus has an outer radius of $2 \times F W H M$. At $353 \mathrm{GHz}$, this corresponds to an aperture radius of about 4.8 and a radius of the outer annulus of 9'.6. This corresponds well to the total field of view $\left(12^{\prime}\right)$ of the entire Laboca bolometer array and should thus give a zero-order approximation of the large-scale spatial filtering. The flux density (APERFLUX) is catalogued as 8.248 $\pm 3.334 \mathrm{Jy}$. Corresponding PSF-fitting photometry (which also takes into account a background offset) is listed as $8.570 \pm 1.214 \mathrm{Jy}$. Applying the same aperture on the Laboca data yields a total flux density of $8.034 \mathrm{Jy}$ which is in good agreement with the Planck flux. We note that in the Laboca data, the background annulus contains flux that is indistinguishable from zero flux, and the entire emission of the core can be contained in a significantly smaller aperture than the one used for Planck.

\subsection{Higher resolution: Extinction mapping}

Since Herschel-based column density maps require a fit to the SED, their angular resolution is limited by the band with the largest beam size. This is the SPIRE $500 \mu \mathrm{m}$ band with a beam size of $36^{\prime \prime} F W H M$. However, when mapping the dust extinction instead of the dust emission, an even better angular resolution can be reached. In our VLT near-infrared data of the FeSt 1-457 core, there are enough background stars to produce a NICEST extinction map with a resolution of $9^{\prime \prime} F W H M$ (with a minimum of about ten background stars per resolution element, see Sect. 2.3). Fitting a 2D Gaussian to the core as a zero-order approximation confirms a slight asymmetry of the core where the larger dimension is $22 \%$ larger than the smaller one: the 2 D Gaussian has widths of 1'.86 $\times 1.52$ FWHM. To show the radial structure of the FeSt 1-457 core, we additionally plot the column density as a function of distance to the peak extinction pixel. Figure 9 shows the resulting profile when based on the infrared extinction profile. No annular averaging has been applied to highlight the slight ellipticity of the core. For comparison, we additionally show a profile from an extinction map with a resolution of $18^{\prime \prime}$. While the profiles look very similar, the slight non-circular structure only becomes clearly apparent in the highresolution extinction map. These high-resolution extinction data of the FeSt 1-457 and other cores will be discussed in more detail by Román-Zúñiga et al. (in prep.).

\subsection{Comparison with Barnard 68}

We briefly test the robustness of our conclusions by applying the same methodology to a second starless core, Barnard 68, which is also located in the Pipe Nebula (Alves et al. 2001). Two recent studies use Herschel submillimeter imaging and subsequent modeling to construct physical models of this core, deriving an absolute central dust temperature of $8.2_{-0.7}^{+2.1} \mathrm{~K}$ or $9.3 \pm 0.5 \mathrm{~K}$ (Nielbock et al. 2012; Roy et al. 2014).

To compare Barnard 68 with FeSt 1-457, we make use of effective dust temperature and dust optical depth maps that are based on the same data as described above. These maps indicate a temperature gradient with a minimum effective dust temperature at the center of Barnard 68 of $13.07 \pm 0.09 \mathrm{~K}$, slightly lower than in the case of FeSt 1-457. The Planck-derived dust opacity spectral index is $\beta_{\text {Planck }}(\mathrm{B} 68)=1.71 \pm 0.06$.

Additionally, we reduced APEX-Laboca science verification observations of Barnard 68 from project 078.F-9012 in the same iterative procedure that we described above for the FeSt 1-457 data. These observations were obtained on July 17, 
2007. For the comparison, the Laboca data were again convolved to a resolution of $36^{\prime \prime}$ (FWHM). The rms noise in the image is at $14 \mathrm{mJy} /$ beam, similar to the case of FeSt 1-457. Finally, we have constructed a near-infrared extinction map from data obtained as part of the same programs that also covered the FeSt 1-457 core.

A comparison of $\tau_{353 \mathrm{GHz}}$ and near-infrared $A_{K}$ for B 68 reveals a linear relation up to the peak value of $A_{K} \sim 3 \mathrm{mag}$ (not shown), with larger scatter due to noise than in the case of FeSt 1-457. There is thus no evidence for a change in $\gamma$ in B 68, but even in the FeSt 1-457 core the change is only becoming apparent at the highest levels of extinction, significantly exceeding the column densities in B 68 .

When comparing the Laboca flux density to the model prediction, we again find a linear function where $a=1.05 \pm 0.01$ and a zeropoint offset of $-0.130 \pm 0.004 \mathrm{Jy} /$ beam. When compared to FeSt 1-457, the zeropoint offset is smaller for this more isolated core, and the slope $a$ is closer to a value of one. The dust opacity spectal index $\beta$ thus is even closer to the Planck value than in the case of FeSt 1-457, with $\beta_{\mathrm{N}}(\mathrm{B} 68)=1.71-0.05=$ $1.66 \pm 0.07$. As a result, the $1 \sigma$ confidence intervals of the dust opacity spectral indices derived for the FeSt 1-457 and B 68 cores overlap.

\section{Summary and conclusions}

The main result of this paper is our finding of evidence for grain growth in the inner regions of the FeSt 1-457 core from a comparison of Herschel-derived dust optical depths with corresponding near-infrared extinction measurements. We conclude that $1 / \gamma$, i.e., the ratio of the submillimeter dust opacity and the nearinfrared extinction coefficient, rises nonlinearly with extinction above about $A_{K}=3 \mathrm{mag}$. The peak extinction corresponds to an estimated average volume density of $2.1 \times 10^{5} \mathrm{~cm}^{-3}$. In the light of previous work (Ascenso et al. 2013), it seems likely that this change is mostly due to an increase of the submillimeter dust opacity $\kappa_{v}$ rather than a decrease of the near-infrared extinction coefficient. No comparable effect was found toward B 68, but this core does not reach the same column densities.

Our second main finding in this paper is that toward the two dense cores FeSt 1-457 and Barnard 68, there is no evidence for variations in the dust opacity spectral index $\beta$ over a wide range of physical scales. A single value of $\beta$ is sufficient to explain the dust emission within both FeSt 1-457 and Barnard 68. There is no evidence for a varying dust opacity spectral index in either core. We find $\beta=1.53 \pm 0.07$ for FeSt $1-457$ and $\beta=1.66 \pm 0.07$ for Barnard 68. The two cores thus have very similar dust opacity spectral indices. These values of $\beta$ are, finally, very similar to the numbers derived in the Planck all-sky dust model in the same locations.

Our experiment thus gives similar results for two different cores (FeSt 1-457 and B 68). Given the number of parameters involved, it would already be a fortuitous coincidence to produce the linearity in Fig. 8 with variations in multiple parameters that would have to cancel out in their effect on $\beta$. Obtaining the same result in a second core underlines the conclusion that the absence of $\beta$ variations on the scales probed is the most simple explanation, involving just a single parameter.

The dust opacity spectral index toward the FeSt 1-457 and Barnard 68 cores thus is essentially the same whether derived on scales of $\sim 1 \mathrm{pc}\left(30^{\prime}\right.$ at a distance of $\left.130 \mathrm{pc}\right)$ or scales of $\sim 0.02$ pc (the Herschel resolution of $36^{\prime \prime}$ ). Using the easily available Planck-derived dust opacity spectral indices would constitute excellent starting points for a study of these cores. This is in notable contrast to Planck-derived effective dust temperatures where the resolution does make a difference and the temperature profile of nearby cores is resolved better using Herschel-based maps. However, the absence of evidence for $\beta$ variations means that reliable effective dust temperature maps can be produced.

Additional results and conclusions of this paper can be summarized as follows:

- The effective dust temperature toward the center of FeSt $1-457$ is $13.5 \mathrm{~K}$ which is higher than the ammonia kinetic gas temperature of $9.5 \mathrm{~K}$, measured toward the same core. This could be due to insufficient thermodynamic coupling of dust and gas, or, more likely, the effect of averaging along the line of sight, or both.

- A comparison of the Herschel effective dust temperature map of FeSt $1-457$ with a map of $\mathrm{N}_{2} \mathrm{H}^{+}$emission shows that this molecular emission is constrained to the coldest and densest parts of the core, confirming previous work on a sample of Pipe cores. Maps of effective dust temperature, which are easier to obtain than millimeter line maps, thus can serve as a reasonable means of identification of the coldest and densest parts of molecular clouds in spite of the line-of-sight averaging.

- There is a zeropoint offset in the Laboca data which is entirely due to spatial filtering of large-scale emission that is unrelated to the core, as can be shown in comparison to Planck data.

- For a detailed study of the spatial structure of nearby cores, the resolving power of near-infrared extinction mapping remains unsurpassed, particularly for targets observed against the Galactic bulge. Based on near-infrared observations that were obtained with the VLT, we present a NICEST extinction map with a resolution of $9^{\prime \prime} F W H M$, or $0.006 \mathrm{pc}$ at the distance of the Pipe Nebula. This is better by a factor of four when compared to Herschel information that is based on a convolution to the resolution of the SPIRE $500 \mu \mathrm{m}$ band.

Acknowledgements. We thank an anonymous referee for comments that helped us to clarify and significantly improve the paper, Ralf Launhardt for insightful discussions, Arnaud Belloche, Giorgio Siringo, and Axel Weiss for help with the Laboca data analysis, and Ted Bergin for providing us with the IRAM data from the Aguti et al. (2007) paper. Herschel is an ESA space observatory with science instruments provided by European-led Principal Investigator consortia and with important participation from NASA. APEX is a collaboration between Max Planck Institut für Radioastronomie (MPIfR), Onsala Space Observatory (OSO), and the European Southern Observatory (ESO). This publication in A\&A is supported by the Austrian Science Fund (FWF).

\section{References}

Aguti, E. D., Lada, C. J., Bergin, E. A., Alves, J. F., \& Birkinshaw, M. 2007, ApJ, 665, 457

Alves, J. F., Lada, C. J., \& Lada, E. A. 2001, Nature, 409, 159

Alves, F. O., Frau, P., Girart, J. M., et al. 2014, A\&A, 569, L1

Ascenso, J., Lada, C. J., Alves, J., Román-Zúñiga, C. G., \& Lombardi, M. 2013, A\&A, 549, A135

Beckwith, S. V. W., \& Sargent, A. I. 1991, ApJ, 381, 250

Belloche, A., Schuller, F., Parise, B., et al. 2011, A\&A, 527, A145

Bianchi, S., Gonçalves, J., Albrecht, M., et al. 2003, A\&A, 399, L43

Draine, B. T., \& Lee, H. M. 1984, ApJ, 285, 89

Forbrich, J., Lada, C. J., Muench, A. A., Alves, J., \& Lombardi, M. 2009, ApJ, 704, 292

Forbrich, J., Posselt, B., Covey, K. R., \& Lada, C. J. 2010, ApJ, 719, 691

Forbrich, J., Öberg, K., Lada, C. J., et al. 2014, A\&A, 568, A27

Frau, P., Girart, J. M., Beltrán, M. T., et al. 2010, ApJ, 723, 1665

Güsten, R., Nyman, L. A., Schilke, P., et al. 2006, A\&A, 454, L13

Kelly, B. C., Shetty, R., Stutz, A. M., et al. 2012, ApJ, 752, 55

Kramer, C., Richer, J., Mookerjea, B., Alves, J., \& Lada, C. 2003, A\&A, 399, 1073

Lada, C. J., Muench, A. A., Rathborne, J., Alves, J. F., \& Lombardi, M. 2008, ApJ, 672, 410 
A\&A 580, A114 (2015)

Lombardi, M. 2009, A\&A, 493, 735

Lombardi, M., Alves, J., \& Lada, C. J. 2006, A\&A, 454, 781

Lombardi, M., Bouy, H., Alves, J., \& Lada, C. J. 2014, A\&A, 566, A45

Nielbock, M., Launhardt, R., Steinacker, J., et al. 2012, A\&A, 547, A11

Ormel, C. W., Min, M., Tielens, A. G. G. M., Dominik, C., \& Paszun, D. 2011, A\&A, 532, A43

Ossenkopf, V., \& Henning, T. 1994, A\&A, 291, 943

Peretto, N., André, P., Könyves, V., et al. 2012, A\&A, 541, A63

Planck Collaboration IX. 2014, A\&A, 571, A9

Planck Collaboration XI. 2014, A\&A, 571, A11

Planck Collaboration XXVIII. 2014, A\&A, 571, A28

Rathborne, J. M., Lada, C. J., Muench, A. A., Alves, J. F., \& Lombardi, M. 2008, ApJS, 174, 396

Román-Zúñiga, C. G., Lada, C. J., \& Alves, J. F. 2009, ApJ, 704, 183
Román-Zúñiga, C. G., Alves, J. F., Lada, C. J., \& Lombardi, M. 2010, ApJ, 725,2232

Roy, A., André, P., Palmeirim, P., et al. 2014, A\&A, 562, A138

Ryter, C. E. 1996, Ap\&SS, 236, 285

Sadavoy, S. I., Di Francesco, J., Johnstone, D., et al. 2013, ApJ, 767, 126

Schnee, S., Kauffmann, J., Goodman, A., \& Bertoldi, F. 2007, ApJ, 657, 838

Schnee, S., Enoch, M., Noriega-Crespo, A., et al. 2010, ApJ, 708, 127

Schnee, S., Mason, B., Di Francesco, J., et al. 2014, MNRAS, 444, 2303

Shetty, R., Kauffmann, J., Schnee, S., \& Goodman, A. A. 2009a, ApJ, 696,676

Shetty, R., Kauffmann, J., Schnee, S., Goodman, A. A., \& Ercolano, B. 2009b, ApJ, 696, 2234

Shirley, Y. L., Nordhaus, M. K., Grcevich, J. M., et al. 2005, ApJ, 632, 982

Siringo, G., Kreysa, E., Kovács, A., et al. 2009, A\&A, 497, 945

Vuong, M. H., Montmerle, T., Grosso, N., et al. 2003, A\&A, 408, 581 